\title{
Designed to Fit: Challenges of Interaction Design for Clothes Fitting Room Technologies
}

\author{
Bo Begole, Takashi Matsumoto, Wei Zhang, Nicholas Yee, Juan Liu, \\ and Maurice Chu \\ Palo Alto Research Center \\ 3333 Coyote Hill Road \\ Palo Alto, CA 94304 \\ bo@parc.com, ma22n@pileus.net, zhangwe@eecs.oregonstate.edu, \\ Nicholas.Yee@parc.com, Juan.Liu@parc.com, Maurice.Chu@parc.com
}

\begin{abstract}
This paper uncovers issues in the design of camera-based technologies to support retail shopping in a physical store, specifically clothes shopping. An emerging class of technology is targeting the enhancement of retail shopping, including the trying on of clothing. Designing such systems requires careful considerations of physical and electronic design, as well as concerns about user privacy. We explore the entire design cycle using a technology concept called the Responsive Mirror through its conception, prototyping and evaluation. The Responsive Mirror is an implicitly controlled video technology for clothes fitting rooms that allows a shopper to directly compare a currently worn garment with images from the previously worn garment. The orientation of images from past trials is matched to the shopper's pose as he moves. To explore the tension between privacy and publicity, the system also allows comparison to clothes that other people in the shoppers' social network are wearing. A user study elicited a number of design tradeoffs regarding privacy, adoption, benefits to shoppers and merchants and user behaviors in fitting rooms.
\end{abstract}

Keywords: Ubiquitous computing, pervasive, ambient intelligence, retail technologies, privacy, online social networks, fashion.

\section{Introduction}

Shopping for clothes is an information seeking activity. Shoppers want information about availability, cost, size, colors, texture, feel, fit, style trends, etc. In contrast to the rapid evolution of the online shopping, the experience of shopping in a physical retail store has changed little. Due to the inherent requirements of physical space, shoppers must expend more energy and time searching for products in a physical environment than when searching online. Physical shopping also provides certain kinds of tactile and physical information that cannot easily be communicated electronically: texture, fit, drape, flow, movement, light refraction, heft, etc. These kinds of information are difficult to communicate electronically because they use human sensing modalities that are not easily quantifiable for electronic transfer and/or are based on each individual's subjective perception. A number of ubiquitous computing 
technologies are providing new capabilities to supplement the physical shopping experience. In addition to displaying conventional product information found on the web, these technologies also augment a human's assessment of the more subjective, perceptual properties by capturing the shopper's experience for use as a memory aid and as an artifact for sharing with friends and family.

This paper explores the design considerations for creating interactive technologies for clothes fitting rooms using the design and evaluation of a prototype technology called the Responsive Mirror (Figure 1) [35, 36, 37], which is an implicitly controlled video technology for clothes fitting rooms that allows a shopper to directly compare a currently worn garment with images from the previously worn garment. The orientation of images from past trials is matched to the shopper's pose as he moves. To explore the tension between privacy and publicity, the system also allows comparison to clothes that other people in the shoppers' social network are wearing.

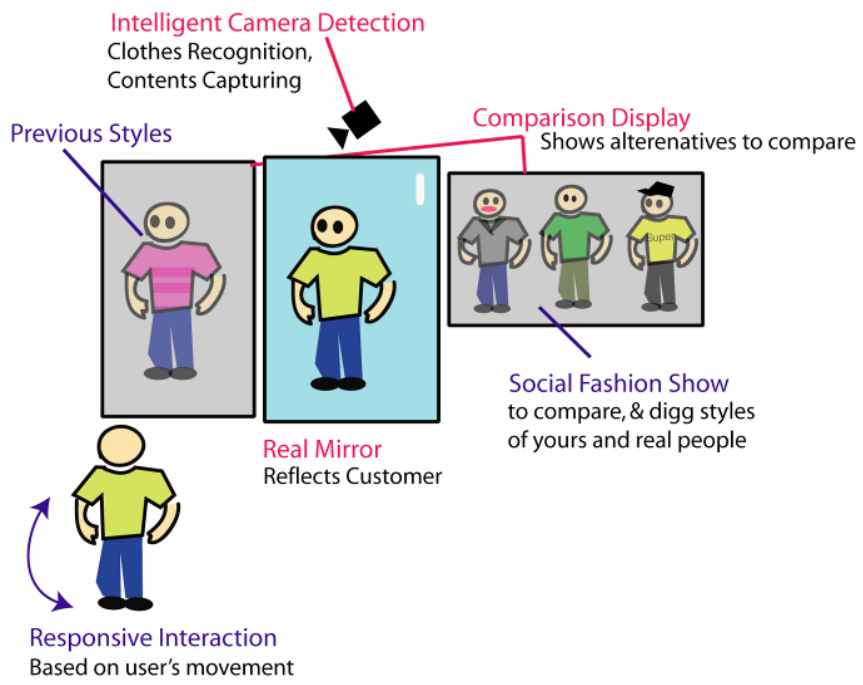

Fig. 1. The Responsive Mirror concept

\section{Related Work}

Clothing stores often provide an area for trying on clothes. Generally, there is a private place in which to change clothing, which we refer to as a changing area, and an area with a mirror to view the fit, which we refer to as a fitting area. In this paper, we consider these areas separately, but they are sometimes combined in one place. The fitting-area mirror is a specific point of customer interaction with products where a shopper selects items to purchase. Marketers have recognized this "point of decision" as an opportunity to engage the customer with supplemental information. 


\subsection{Clothes-Fitting Technologies}

Technologies in Fitting Rooms: A Prada boutique in New York contains a sophisticated dressing room designed and constructed by Ideo and IconNicholson [16]. A scanner identifies each garment as the shopper takes it in, providing additional information about the garment's price and alternate colors and sizes - the kind of information shoppers can find when browsing products online. The fitting room also contains a motion-triggered video camera that records the shopper and plays back the video after a pause.

One of the central components of the system is the Magic Mirror which allows a person trying on clothes to send video of himself to friends who can send back comments and vote (thumbs up/down) [21]. The Magic Mirror can also project a static image of an alternate garment onto the mirror allowing the person to see roughly how it might look on him.

The Magic Mirror has also been deployed in Bloomingdales. Similar systems have been created and deployed for market evaluation in a number of other retailers [34]. The Gardeur Shop in Essen Germany created an RFID-based system that provides product information including price, available sizes and colors, material and care instructions. Warnaco created a system to show fitting information for women's braziers. Poggi reports other retailers employing non-sensor technologies in fitting rooms, such as Metropark, Nordstrom, Kira Plastinina and Macy's.

Virtual Fittings: Some online services, such as Intellifit [14] and MyShape [19], take measurements of a customer's body and suggest clothes that will fit and provide a flattering appearance for that body shape. These services have been deployed in stores such as Levi's and Charming Shoppes in Ohio, Florida and Texas [34]. Other web services such as MyVirtualModel [20], Knicker Picker [17], and 3Dshopping [1] provide a set of predefined body types that allow a shopper to view the fit of clothes on their selected body type. It is also possible to use augmented reality techniques to map an image of a garment onto a captured image of the customer that will change shape in real time as the customer moves in a virtual mirror [8].

In essence, these virtual fitting technologies are comparable to seeing clothing on a personalized mannequin. However, just as one needs to try on clothes seen on a mannequin, a customer must try the garment on his own body to get the full experience of feel, drape and look. Although virtual fitting technologies continue to advance, Protopsaltou et al. report that reliance on such technology often results in unexpected fit or color, and unpleasant feel of the fabric [25]. Such technologies also do not cover the full range of ways in which a person can wear a garment. A buttoned down shirt, for example, can be worn buttoned or unbuttoned, tight or baggy, tucked or untucked, tied at the bottom, and sleeves rolled to different lengths. Exploring this variety of options ultimately requires physical control of the clothes.

\subsection{Reactive Displays}

The ability to change information in reaction to the presence and motion of people has been demonstrated in several prior systems. Haritaoglu and Flickner describe a prototype that uses computer vision to count the people looking at a display and to infer demographic information [13]. There are some commercial advertising technologies 
(Reactrix [26], Freeset Human Locator [10], P.O.P. ShelfAds [24]) that detect presence and motion of people and change the content of the display in reaction. These reactive displays do not display images that mirror the image or motion of the person looking at the display as the Responsive Mirror does.

\subsection{Privacy Concerns from Cameras in a Fitting Room}

A common concern that sensor technologies face is how they affect a user's sense of personal privacy. Will users accept a camera or other sensors into a traditionally semiprivate space such as a fitting area? What concerns would they have and what measures should the system design incorporate to mitigate such concerns? In the press articles, privacy concerns are sometimes raised but rarely explored in depth.

People are subjected to video capture on a daily basis, sometimes happily (home movies, video conferencing, camera-based game controllers) and sometimes without much choice (store surveillance, toll collection, traffic cameras). Such applications have achieved at least some level of tolerance, yet debate continues about the overall effects of sensing technologies on the long-term health of our society. Grudin [12] observes that collecting and distributing such data electronically heightens concerns of being susceptible to undesirable uses. A basic framework of feedback and control of image capture was outlined by Bellotti and Sellen [5], which systems such as the Responsive Mirror commonly employ. Nevertheless, when a camera or other contextsensing technology is introduced into an unexpected situation, such as a fitting room, we need to examine the potential impact on privacy.

Palen and Dourish [22], synthesizing Irwin Altman's research of face-to-face interaction [2], describe the boundaries and tradeoffs between publicity and privacy that people navigate under technology-mediated interactions. Systems such as the Responsive Mirror introduce new capabilities that affect people's expectations under the three boundaries of their framework, described next. Also relevant is the work of Petronio [23] who examines a multitude of publicity/privacy boundaries and the varying permeability of the boundaries. We describe below the potential encroachments across these boundaries that camera technologies create.

Disclosure: People naturally have an expectation that a fitting room is a private place where they can change clothes without being seen. Although the Responsive Mirror cameras do not capture images of people changing clothes in the changing area itself, only in the fitting area outside, an individual may still have some concern of undesired disclosure of potentially embarrassing interactions in the mirror. In addition to the awkward poses that a shopper may take to test the fit of clothing, a shopper naturally expects that she can experiment with clothes styles in which she might not like to be seen for any number of reasons: fit, style, status, expressiveness, etc. She may be troubled if the images were seen by other people.

On the other hand, a shopper interacting with Responsive Mirror may not desire that he fittings be completely undisclosed. She may want to show clothes to friends, or publish them to a social fashion network.

Identity: The Responsive Mirror presents images of the shopper as well as others while making decisions about clothing that will become part of his presentation of self. The ability to see images of others provides a means for a person to test the fit of 
his presentation of self among the presentations of a community. He can examine styles that others are wearing and choose where he would like to fit. On the other hand, he may not desire to be seen as actively positioning himself along such dimensions, perhaps preferring to present a nonchalant attitude about fashions. Thus, a record of his examinations of the images of other people could be sensitive.

Temporal: Shoppers today expect that an experimental fitting is transient, not lasting for others to see at another time. In addition to the obvious concern that a shopper might have about recoding images in an embarrassing outfit, images in flattering outfits could become a concern in the future as style and tastes change. While a flattering garment may be an excellent choice at the moment, it could come to represent something contrary to the shopper's style in the future. This concern can be mitigated by providing the ability to delete regrettable images, but the damage cannot be wholly undone as the images may have been stored in an Internet archive. Furthermore, simple deletion is possibly more drastic than people would desire. The temporal boundary is one that has a varying permeability over time and people might prefer simply that it become more difficult, but not impossible, to see older images.

Social Aspects of Fashion Decisions: Another under-explored consideration for such technologies is the social aspects of shopping and fashion. Only the Magic Mirror begins to provide support for social interactions by employing mechanisms for synchronous image sharing and voting by remote friends or family. These capabilities are only the tip of the iceberg regarding social dynamics of shopping and clothing. What kind of social impressions are shoppers concerned about? How do such systems support exploration of the "language" of clothing?

When buying clothes, shoppers often assess how others would perceive a particular item, that is, the "fashion statement" she is making. Indeed, people often ask companions or store assistants how they look to get this kind of feedback. Although evaluating one's self image is an important part of clothes-buying decisions, shoppers in a store are provided very little information about what other people are wearing, other than what is presented in advertisements. Outside of the store, people find inspiration from others and from fashion media, some of which today contain photographs of amateur people.

Sociologists have long explored the multiple roles that fashion plays in society. In a synthesis of more than 200 sources, Davis [7] examines the social construction of meaning in fashion and how an individual's choices communicate social status, gender identity, sexuality, and conformity among other characteristics. Clothing is undoubtedly a form of communication, as declared famously by Lurie [16] and examined in depth by Barnard [4], but Davis believes it is a code of "low semanticity" because the meanings differ with time, trends and among different individuals.

Although technologies cannot interpret fashion, they can mediate human discourse on the topic. We see this happening today in online social fashion networks, such as ShareYourLook [28] and IQONS [11, 15]. These sites allow members to upload photographs of themselves wearing various outfits and they can categorize, tag and comment on the images. The services merely provide scaffolding; the members construct the semantics through tagging and commentary. Providing access to a shopper's social fashion network during a shopping experience would provide access to the state of fashion as constructed by the shopper and her online community. 


\section{Formative Evaluation of Responsive Mirror}

Our prototype was designed to test assumptions about the usefulness of the new capabilities and to draw out lessons for future design. First, will shoppers benefit from being able to directly compare their clothing alternatives in a physical shopping experience? Second, will shoppers find value from images of other people wearing similar and different clothes? We also wanted to learn which, if either, feature would shoppers prefer, what uses they would make of them and what other capabilities they would desire. We considered conducting field observations in an actual store's fitting room but determined that before doing so we needed to gain some understanding of the privacy implications of the camera. Therefore, in this initial study, we observed people's behavior using the prototype in our lab.

We recruited 12 male participants who wear medium to large men's shirt size in the age range of 28-52 years old. We were only able to use male participants because the prototype's body segmentation and clothes detection algorithms are initially targeted for men's shirts because there is less variance among men's shirt varieties than among women's. We recognize that men are only a sub-population of the eventual user population but testing on this demographic allowed faster iteration of the technology-design cycle. A limitation of this study is that it used participants of only one gender (male). Thus, it is not clear to what extent the same phenomena would be observed with participants of the other gender (female), particularly regarding questions of privacy and social effects.

The experiment consisted of three conditions.

1. Mirror: Conventional mirror alone.

2. Previous Outfit: Conventional mirror with a display on the left showing an image of the person from the most recent previous fitting. The orientation of the person in the image matches the orientation of the person in realtime.

3. Other People: Conventional mirror with a display on the right showing four images of other people.

For each condition, participants put on six different shirts consisting of three collared polo-style shirts and three crew neck shirts of a mix of colors. The price of the shirt was removed. There were three sets of six shirts and there was a comparable mix of colors and patterns across the sets. The order of conditions and shirt sets was counter-balanced using a latin-square three-by-three design.

\subsection{Task and Procedure}

Participants were told to imagine themselves in a scenario where they need to decide which, if any, of the six shirts they will buy. To eliminate price as a factor in their decision, participants were told to imagine that they can afford as many shirts as they like and would consider the prices to be reasonable in all cases.

For each shirt, the participant stood approximately $250 \mathrm{~cm}(8.2 \mathrm{ft})$ in front of a fulllength mirror ( $165 \mathrm{~cm}$ ( 65 inches) high by $73 \mathrm{~cm}$ ( 29.5 inches) wide). Participants were asked to view the shirt from multiple angles in a natural way. After trying all six shirts per condition, the participants were asked to fill out a questionnaire measuring how appealing they found the shirt and how likely they are to buy the shirt on a 5 point 
scale. They were also asked questions about the condition they had just experienced. After all three conditions were complete, the participants filled out a questionnaire with questions spanning the conditions and potential privacy issues.

\subsection{Results}

To examine whether the display conditions affected participants' perception of the appeal or desire to purchase any of the shirts, we conducted two repeated-measures ANOVAs with Display Condition as the within-subject variable, and the appeal and purchase variables as dependent variables in turn. Neither the ratings of the desire to purchase nor appeal of the shirts were significantly different among the conditions (respectively $\mathrm{F}[2,110]=.42, \mathrm{p}=.66$ and $\mathrm{F}[2,110]=.32, \mathrm{p}=.73$ ).

We asked participants to rank their preference for the three conditions plus a configuration using all three components (which they did not experience). To test whether participants ranked these four configurations differently, we performed a Friedman test on the ranked values within subjects. There was a significant difference in the rankings $\left(\chi^{2}=9.10, \mathrm{p}=.03\right)$, where the preference (from strongest to weakest) were: Previous Outfit plus Other People $(M=1.92)>$ Previous Outfit $(M=2.00)>$ Other People $(\mathrm{M}=2.83)>$ Plain Mirror $(\mathrm{M}=3.25)$.

And finally, we asked participants how much the Previous Outfit and Other People conditions enhanced their shopping experience. Participants rated the Previous Outfit $(\mathrm{M}=3.00, \mathrm{SD}=.85$, where $5=$ Extremely Helpful and $1=$ Not Helpful At All $)$ as having enhanced their experience more than the Other People $(\mathrm{M}=2.50, \mathrm{SD}=.52)$ condition, which is in line with the ranked measures. A paired-samples t-test showed the ratings to be significantly different between the conditions $(\mathrm{t}[11]=-2.57, \mathrm{p}=.03)$.

\subsection{Results from Privacy Related Questions}

Regarding the issues of disclosure, we asked participants how much it would bother them if someone from same or opposite gender and specific social group (family, friends, co-workers, and stranger) viewed images of them that were captured by the system. They specified their response on a five point scale $(5=$ bothers me a great deal, $1=$ doesn't bother me at all). The responses were not significantly different according to the gender of who might see the image, so we calculated the mean for each social group across gender. The means were Family $=1.08(\mathrm{SD}=.19)$, Friends $=1.50(\mathrm{SD}=$ $.71)$, Stranger $=2.08(\mathrm{SD}=1.06)$ and $\mathrm{Co}$-worker $=2.25(\mathrm{SD}=1.14)$. We performed a repeated-measures ANOVA and found there was a significant difference among the social groups $(F[3,33]=5.76, p=.003)$. Post-hoc contrasts revealed that Family is not significantly different from Friends. and that CoWorkers is not significantly different from Strangers. Family and Friends are significantly different from CoWorkers and Strangers $(\mathrm{p}=.01)$. The frequency distributions for all groups have a mode of 1 (doesn't bother me at all) except Coworkers which is bimodal with modes at 1 (doesn't bother me at all) and 3 (bothers me somewhat).

We asked participants how much it would bother them on the same 5 point scale if other people saw images of them wearing a shirt that looks good versus a shirt that looks bad on them. Participants rated their level of concern significantly higher for bad shirts $(\mathrm{M}=3.0)$ versus good shirts $(\mathrm{M}=1.42)(\mathrm{p}=.001)$. 
Regarding the aspects of personal and group identity, we asked participants how often (5=Always, Often, Sometimes, Seldom, Never=1) they think of someone else who might like the clothes they are trying on. The mean response was 2.67 $(\mathrm{SD}=0.98)$. We asked participants how often they think about how similar the clothes are to what other people they know and don't know are wearing. The mean for people they know was $2.92(\mathrm{SD}=0.9)$ and for people they don't know was 2.33 $(\mathrm{SD}=0.98)$ with no significant difference between them. Participants responded with a mean of $3.6(\mathrm{SD}=1.07)$ to the question of how often do they consciously consider how others will perceive them in the clothes they are trying.

Regarding the issues of temporality, we asked participants if their tastes changed in the future, would they want to remove images of themselves wearing a contrary style. On a five point scale (5=Definitely, $1=$ Definitely Not), participants responded with a mean of 3.08 ( $\mathrm{SD}=1.16)$ (closest to Possibly). We asked participants at what period of time would they consider removing images of themselves that they had allowed others to see. The highest number of responses was for 3 months (5 participants) and the distribution of the remaining responses was spread across times within 1 year (5 participants) and never ( 2 participants). These responses can guide the points in time at which to remind users of the existence of past images.

\section{Lessons for Design}

Our experience with the Responsive Mirror uncovered some unexpected issues in the design of user experience in this domain.

Because of the error rates of sensing technologies, designers need to anticipate variance in the user experience. Our prototype used a straightforward color-histogram comparison from a relatively small data set and yet we found that sometimes the system retrieved different matches for different participants wearing the same clothes. Although this inconsistency was not noticed by the individual participants, designers need to be aware of potential variance in system behavior and avoid designs that expect a deterministic result.

Designers of pervasive technology must be aware of the ways that information systems such as these are affected by our notions of personal privacy. We already see that the increasing volumes of social content creation, sharing and retrieving are modifying societal norms. Designers of retail systems should shape the system to provide benefits that balance, or potentially outweigh, the risks to privacy in terms of disclosure, identity and temporal boundaries. Our experience indicates that some users are receptive to a system that bundles the capture of potentially sensitive images with the benefit of seeing images of self and others.

Perhaps most important to merchants, our results showed no difference in participants' perceptions of their likelihood to buy a shirt after using our prototype. The quality of the product matters more than the technologies used to evaluate it. Nonetheless, participants did perceive the system to have been "helpful" to their buying decision, providing value that merchants should be able to capitalize on.

Pervasive technologies such as the Responsive Mirror change the clothes-buying experience by connecting information between our physical lives and online digital identities. Currently these realms are artificially separated in a false dichotomy of 
"virtual" versus "real" life. Context-sensing technologies enable the design of applications such as Responsive Mirror that not only augment the immediate physical experience but also integrate information across the multiple aspects of one's social life. As we see increasing spread of such technologies, designers need to be informed of the unique considerations that must be made in these environments, particularly with respect to privacy and social goals.

\section{References}

1. 3DShopping.com, http://www.3dshopping.com

2. Altman, I.: The Environment and Social Behavior: Privacy, Personal Space, Territory and Crowding. Brooks/Cole Pub. Co., Inc., Monterey (1975)

3. Anguelov, D., Lee, K., Gokturk, S.B., Sumengen, B.: Contextual identity recognition in personal photo albums. Computer Vision and Pattern Recognition (CVPR) (2007)

4. Barnard, M.: Fashion as Communication, 2nd edn. Routledge (2002)

5. Bellotti, V., Sellen, A.: Design for privacy in ubiquitous computing environments. In: Proceedings of the European Conference on Computer-Supported Cooperative Work, ECSCW, pp. 77-92. Kluwer Academic Publishers, Norwell (1993)

6. Brown, J.: Prada Gets Personal, BusinessWeek. The McGraw-Hill Companies, March 18 (2002),

http://www.businessweek.com/magazine/content/02_11/ b3774612.htm

7. Davis, F.: Fashion, Culture and Identity. University Of Chicago Press (1994)

8. Ehara, J., Saito, H.: Texture overlay onto deformable surface for virtual clothing. In: Proceedings of the 2005 international Conference on Augmented Tele-Existence ICAT 2005, vol. 157, pp. 172-179. ACM Press, New York (2005)

9. Ehrlich, S.F.: Strategies for encouraging successful adoption of office communication systems. ACM Trans. Inf. Syst. 5(4), 340-357 (1987),

http: / / doi.acm.org/10.1145/42196.42198

10. Freeset Human Locator, http: / / www. freeset.ca/locator /

11. Geller, M.: Friendly fashion - Cyber-style!, Reuters (April 28, 2007), http: //blogs.reuters.com/2007/08/28/ friendly-fashion-cyber-style/ (Last accessed January 29, 2009)

12. Grudin, J.: Desituating Action: Digital representation of Context. Human-Computer Interaction 16(2-4), 269-286 (2001)

13. Haritaoglu, I., Flickner, M.: Attentive billboards. In: Proceedings of the Conference on Image Analysis and Processing 2001, September 26-28, 2001, pp. 162-167. IEEE Press, Los Alamitos (2001)

14. Hilsmann, A., Eisert, P.: Deformable object tracking using optical flow constraints. In: 4th European Conf. on Visual Media Production, 2007, November 27-28, 2007. IETCVMP, pp. 1-8 (2007)

15. Intellifit, http://www. intellifit.com/Intellifit/Home.aspx

16. IQONS, http://www. iqons.com/

17. KnickerPicker.com, http://www. knickerpicker.com/

18. Lurie, A.: The Language of Clothes, Random House (1981)

19. Like.com, http: / /www. like.com/ (Last accessed September 8, 2007)

20. Lou, H., Luo, W., Strong, D.: Perceived critical mass effect on groupware acceptance. Eur. J. Inf. Syst. 9, 91-103 (2000) 
21. MyShape, http: / /www . myshape.com/

22. My Virtual Model, http: / / www . mvm. com

23. Nanda, S.: Virtual Mirrors. Reuters, January 29 (2007), http: / /www.reuters.com/news/video/videoStory?videoId=5219

24. Palen, L., Dourish, P.: Unpacking "privacy" for a networked world. In: Proceedings of the SIGCHI Conference on Human Factors in Computing Systems. CHI 2003, pp. 129-136. ACM Press, New York (2003), http://doi.acm.org/10.1145/642611.642635

25. Petronio, S.: Boundaries of Privacy: Dialectics of Disclosure. State University of New York Press, Albany (2002)

26. P.O.P. ShelfAds, http://www.popbroadcasting.com/main/intilashelf . html

27. Protopsaltou, D., Luible, C., Arevalo, M., Magnenat-Thalmann, N.: A body and garment creation method for an Internet based virtual fitting room. In: Computer Graphics International 2002 Conference Proceedings, pp. 105-122. Springer, Heidelberg (2002)

28. Reactrix, http://www.reactrix.com/advertisers_details.php?id=9

29. RockYou, http: / / www. rockyou.com/ (Last accessed September 8, 2007)

30. ShareYourLook, http://www. shareyourlook.com/ (Last accessed September 3, 2007)

31. Song, Y., Leung, T.: Context-aided human recognition - clustering. In: Leonardis, A., Bischof, H., Pinz, A. (eds.) ECCV 2006. LNCS, vol. 3953, pp. 382-395. Springer, Heidelberg (2006)

32. Weiser, M.: The computer for the 21 st century. Scientific American 265, 94-104

33. Staedter, T.: Smart' Fitting Room Suggests What to Wear. Discovery News, January 23 (2008)

34. Poggi, J.: Dressing Rooms Of The Future, Forbes.com, July 22 (2008)

35. Chu, M., Begole, B.: Human-Centric Interfaces for Ambient Intelligence. In: Aghajan, H., Delgado, R.L.-C., Augusto, J.C. (eds.) Natural and implicit information seeking cues (to appear)

36. Zhang, W., Matsumoto, T., Liu, J., Chu, M., Begole, B.: An intelligent fitting room using multi-camera perception. In: Proceedings of the 13th international Conference on intelligent User interfaces (IUI 2008), pp. 60-69. ACM, New York (2008)

37. Zhang, W., Begole, B., Chu, M., Liu, J., Yee, N.: Real-Time Clothes Comparison Based on Multi-View Vision. In: Proceedings of ACM/IEEE International Conference on Distributed Smart Cameras (ICDSC 2008), September 7-11 (2008) 